\title{
Comparison of four channelled videolaryngoscopes to Macintosh laryngoscope for simulated intubation of critically ill patients: the randomized MACMAN2 trial
}

\author{
Paul Decamps ${ }^{1}$, Nicolas Grillot ${ }^{2,3}$, Aurelie Le Thuaut ${ }^{4}$, Noelle Brule ${ }^{1}$, Corinne Lejus-Bourdeau ${ }^{2,3}$, \\ Jean Reignier ${ }^{1}$ and Jean-Baptiste Lascarrou ${ }^{1,5^{*}}$ (i)
}

\begin{abstract}
Background: Videolaryngoscopes with an operating channel may improve the intubation success rate in critically ill patients. We aimed to compare four channelled videolaryngoscopes to the Macintosh laryngoscope used for intubation of a high-fidelity simulation mannikin, in a scenario that simulated critical illness due to acute respiratory failure.

Results: Of the 79 residents who participated, 54 were considered inexperienced with orotracheal intubation. Each participant used all five devices in random order. The first-pass success rate was 97.5\% [95\% Cl 91.1-99.7] for Airtraq ${ }^{\mathrm{TM}}$, KingVision $^{\mathrm{TM}}$, and Pentax AWS200 ${ }^{\mathrm{TM}}$, 92.4\% [95\% Cl 84.2-97.2] for VividTrac VT-A100 ${ }^{\mathrm{TM}}$, and 70.9\% [95\% Cl 59.6-80.6] for direct Macintosh laryngoscopy. The first-pass success rate was significantly lower with direct Macintosh laryngoscopy than with the videolaryngoscopes ( $p<0.0001$ for Airtraq ${ }^{\mathrm{TM}}$, KingVision ${ }^{\mathrm{TM}}{ }$, Pentax AWS200 ${ }^{\mathrm{TM}}$, and VividTrac VT-A100 ${ }^{\mathrm{TM}}$ ).

Conclusion: The Airtraq ${ }^{\mathrm{TM}}$, KingVision ${ }^{\mathrm{TM}}$, and Pentax AWS200 ${ }^{\mathrm{TM}}$ channelled videolaryngoscopes produced high first-pass success rates with a lower boundary of the $95 \% \mathrm{Cl}$ above $90 \%$. A multicentre, randomised controlled clinical study comparing channelled videolaryngoscopy to direct laryngoscopy should include one of these three videolaryngoscopes.
\end{abstract}

Keywords: Intensive care, Macintosh laryngoscope, Videolaryngoscope, Endotracheal intubation, High-fidelity simulation

\section{Introduction}

Endotracheal intubation (ETI) in critically ill patients is a risky procedure associated with serious complications in up to $25-40 \%$ of cases [1-3] and with cardiac arrest in $1.6-2.7 \%$ of cases [4]. The main determinant of complications is failure of the first ETI attempt. The number of ETI attempts, i.e., of laryngoscopies, correlates with the risk of serious complications [5-7]. Difficult ETI defined

\footnotetext{
*Correspondence: jeanbaptiste.lascarrou@chu-nantes.fr

${ }^{5}$ Médecine Intensive Réanimation, Centre Hospitalier Universitaire de Nantes, Nantes, France

Full list of author information is available at the end of the article
}

as two failed orotracheal intubations under laryngoscopy guidance is far more common in the intensive care unit (ICU) than in the operating room, with reported frequencies ranging from 10 to $20 \%$ [8-11].

Videolaryngoscopes were developed to facilitate orotracheal intubation. These devices allow complete indirect vision of the glottis even when the oro-pharyngo-laryngeal axis is misaligned. Although their role in the intensive care unit (ICU) remains unclear [12], they are now available in about $75 \%$ of ICUs $[13,14]$. However, their use as the first-line ETI tool ranges from over $90 \%$ of cases in the US [15] to less than 5\% of cases in France [13]. This variability is related to the absence of convincing 
evidence that videolaryngoscopy improves first-attempt success rates in the ICU: several studies compared firstline videolaryngoscopy to first-line direct laryngoscopy using a Macintosh blade [10, 16-19], but meta-analyses of their findings were inconclusive [20-22]. In the MACMAN1 randomised controlled trial of videolaryngoscopy vs. Macintosh direct laryngoscopy [10], the main cause of ETI failure in the videolaryngoscopy group was glottis catheterisation failure (70.7\%), which occurred despite clear and complete visualisation of the glottis. Videolaryngoscopes that have an operating channel to guide the tube into the trachea, of which several models are available, should reduce the risk of catheterisation failure. However, few studies have compared the various models, and most of them were done in patients undergoing elective surgery or using low-fidelity dummies. The Pentax AWS200 ${ }^{\mathrm{TM}}$ and KingVision ${ }^{\mathrm{TM}}$ proved more efficient than the standard videolaryngoscope during ETI for cardiac arrest [23, 24], whereas another study found no significant differences [25]. The A.P. Advance ${ }^{\mathrm{TM}}$ Difficult airway blade was inferior to direct laryngoscopy and to other videolaryngoscopes in a study of elective-surgery patients equipped with a collar to simulate a difficult airway [26]. This variability in results is probably ascribable to differences in device design.

The objective of this randomised controlled trial (MACMAN 2 trial) was to evaluate four channelled videolaryngoscopes (KingVision ${ }^{\mathrm{TM}}$, Airtraq $^{\mathrm{TM}}$, VividTrac VTA$100^{\mathrm{TM}}$, and Pentax AWS200 ${ }^{\mathrm{TM}}$ ) in a realistic ICU scenario of orotracheal intubation on a high-fidelity mannikin. Our main hypothesis was that each channelled videolaryngoscope had a lower 95\% confidence interval boundary greater than $90 \%$.

\section{Methods}

\section{Design}

The MACMAN 2 trial used a randomised, open-label, single-centre design. Orotracheal intubation was performed on a high-fidelity simulator. The study was registered on As predicted before inclusion of the first participant (\#15045).

\section{Participants}

Recruitment was carried out among anaesthesia and intensive-care residents in their first and second years, emergency-medicine residents in their first to third years, and medical specialty residents who had completed at least one ICU rotation. The participants were contacted by e-mail via existing mailing lists for the different specialties.

The participants were categorised as experienced or inexperienced based on whether they had performed
50 or more successful ETIs using direct laryngoscopy. Before the study, all participants attended a theoretical class that included an 8-min video explaining the study objective and describing the four videolaryngoscopes and their modalities of use according to the manufacturers' recommendations. The video also showed ETI using a laryngoscope equipped with a Macintosh blade.

\section{Intubations on the high-fidelity simulator}

The orotracheal protocol has been described elsewhere $[10,27]$. It consisted of a realistic scenario of ETI in an ICU patient with acute respiratory failure. The study participants performed ETIs on a high-fidelity simulator (SimMan3G ${ }^{\mathrm{TM}}$, Laerdal Medical, Stavanger, Norway). One of the investigators (i.e., an intensivist with extensive ETI experience) was also present and played the role of the on-duty supervising senior intensivist. ETI was made more difficult by inflating the tongue to reduce the size of the pharynx, thereby impairing visualisation of the glottis.

Pre-oxygenation was ensured by a self-refilling balloon with a unidirectional valve (bag-valve-mask, BVM) connected to wall oxygen and fed by a minimum of $15 \mathrm{~L} / \mathrm{min}$ for a minimum of $3 \mathrm{~min}[28,29]$. Fictitious anaesthetic induction was then performed by combining a hypnotic agent (etomidate) and a neuromuscular blocking agent (succinylcholine), in accordance with international [30] and French [31] guidelines.

Laryngoscopy was performed using the randomly assigned device (Fig. 1). The five devices were KingVi$\operatorname{sion}^{\text {TM }}$ (AMBU, Bordeaux, France), AWS200 ${ }^{\mathrm{TM}}$ (PENTAX, Argenteuil, France), Airtraq ${ }^{\mathrm{TM}}$ (VYGON, Ecouen, France), VividTrac VT-A100 ${ }^{\mathrm{TM}}$ (VIVID, Palo Alto, CA), and a Macintosh size 3 blade for direct laryngoscopy. A size 8 PORTEX $^{\mathrm{TM}}$ tube (Smiths Medical France, Rungis, France) was introduced. If intubation proved difficult, the participant could ask for a bougie, without this being considered an intubation failure. The tube balloon was inflated then manually ventilated using a BVM. The intra-tracheal position of the tube was established based on the capnography curve over more than three breathing cycles.

If the first ETI attempt failed, i.e., if the videolaryngoscope or Macintosh blade was removed from the mouth of the mannikin without the tube being in the trachea, the investigator encouraged the participant to try again. The participant could choose to request a backwards, upwards, and rightwards pressure (BURP) manoeuvre or to use a bougie for this second attempt. BVM insufflation was not permitted before or after the first laryngoscopy even if desaturation occurred, because the mannikin was not able to detect successful intubation after BVM insufflation. 


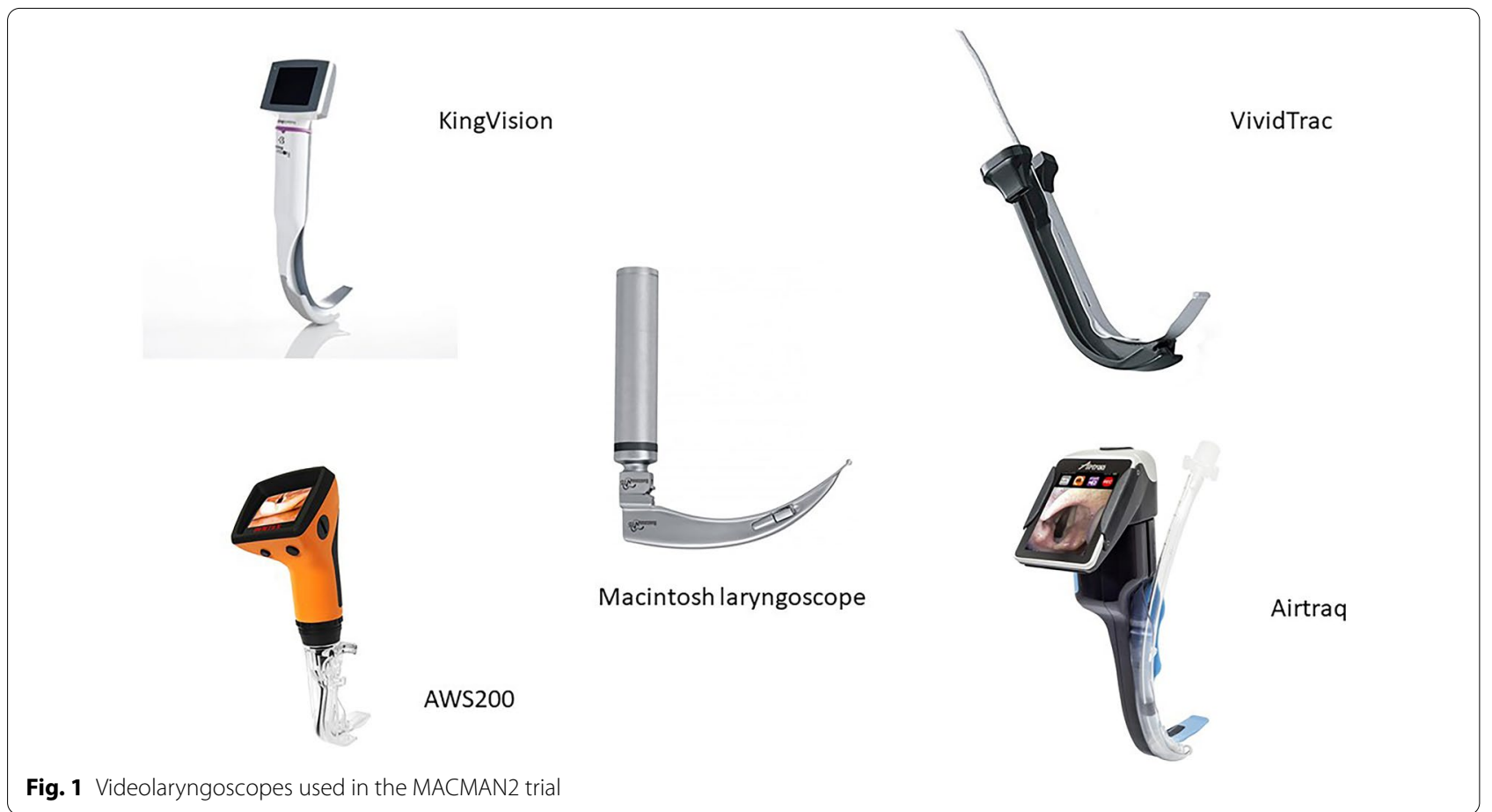

Laryngoscopy duration was defined as the time from the beginning of mannikin fasciculations to confirmation of the intratracheal position of the tube based on the first inflection of the expired $\mathrm{CO}_{2}$ curve. The desaturation model used was constructed from clinical monitoring data collected during the MACMAN study [10].

The participant then repeated this procedure using the other four devices in the order assigned at random. The simulation scenario was re-started at the beginning of the use of each device.

At the end of the session the participant was asked to rate the ease of use of each device.

\section{Randomisation}

Before each session for each participant, the order in which the five devices were used was drawn at random by the investigator by interrogating a dedicated website (https://nantes-lrsy.hugo-online.fr/EnnovClinical/). The randomisation sequence was built by a statistician who was not otherwise involved in the trial (ALT). Blinding was not feasible.

\section{Outcomes}

The primary outcome was the first-pass success rate with each device [32]. ETI failure was defined as oesophageal intubation or removal of the videolaryngoscope or Macintosh blade from the mouth of the mannikin.

The secondary outcomes were time to successful intubation, the Cormack-Lehane and POGO glottis visibility scores, the proportion of attempts resulting in oesophageal intubation, the proportion of attempts resulting in selective bronchial intubation, the median lowest $\mathrm{SpO}_{2}$ value computed from the desaturation model, and ease of device use rated by the participants on a scale from 0 (not at all easy) to 10 (very easy).

\section{Ethics}

The study protocol was approved by the Ethics Committee of the Société de Réanimation de Langue Française (CE SRLF 17-11). Each participant was informed at the beginning of each session about the objectives of the trial, the recording of the simulation sessions, and her or his right to access and refuse the use of the data collected for the trial.

\section{Statistics}

Based on a previous study, we defined a $90 \%$ first-attempt success rate as the lowest clinically acceptable rate for a device designed for the management of difficult airways [26]. We, therefore, defined the target first-attempt success rate as $95 \%$ for the channelled videolaryngoscopes, with a lower boundary of the $95 \%$ confidence interval ( $95 \% \mathrm{CI}$ ) of $90 \%$ or more. With the alpha risk set at $5 \%$, to obtain $80 \%$ power we needed 73 intubations using each device. To account for possible missing data, we decided to include 80 intubations per device.

The statistical analysis was performed using SAS ${ }^{\circledR}$ software (SAS Institute, Cary, NC) with the intention-to-treat 
approach (each intubation was analysed in the group assigned at random). The participants were described by the number and percentage of each modality for qualitative variables and by the mean $\pm \mathrm{SD}$, range, and quartiles for quantitative variables.

To assess the primary outcome, we chose the Chisquare test to compare the five groups.

The secondary outcomes were evaluated using mixed generalised linear or logistic regression models depending on the variable type.

$P$ values were considered significant if $<0.05$ for the primary outcome. For the secondary outcomes, Bonferroni's correction was applied to the comparisons of the four videolaryngoscopes to the Macintosh laryngoscope, and significant $p$ values were $<0.006$.

\section{Results}

Between September 2017 and March 2020, we included 79 residents (Additional file 1: Figure S1), including 40 (51\%) in intensive care or anaesthesiology (Table 1). Of the 79 residents, 75 (96\%) had already performed at least one ETI on a patient by direct laryngoscopy with a Macintosh blade (75 out of $79 ; 96 \%$ ) and 64 (81\%) had already used a videolaryngoscope on a patient. The criterion for being an experienced intubator was met by 25 (32\%) participants.

\section{Primary outcome}

The first-attempt success rate was $97.5 \%$ (95\% CI 91.199.7) for Airtraq, KingVision, and Pentax; 92.4\% [95\% CI 84.2-97.2] for VividTrac; and 70.9\% [95\% CI 59.6-80.6] for direct laryngoscopy. The lower boundary of the $95 \%$ CI was greater than $90 \%$ for three devices, the exceptions being VividTrac and direct laryngoscopy.

\section{Secondary outcomes \\ First-pass success}

There were no significant differences between the firstattempt success rate with VividTrac and with each of the other three videolaryngoscopes ( 92.4 vs. 97.5\%; $p=0.18, p=0.20$, and $p=0.38$, respectively; Fig. 2). The first-attempt success rate for direct laryngoscopy was significantly lower compared to those for the Airtraq, KingVision, and Pentax AWS200 (70.9 vs. 97.5\%; $p<0.0001$; Fig. 2) and to that for the VividTrac (70.9 vs. $92.4 \% ; p<0.0001)$. Experienced and inexperienced intubators obtained similar results (Additional file 2: Figure S2; Additional file 3: Figure S3).

\section{Intubation time}

The laryngoscopy time was longer with direct laryngoscopy than with the Airtraq, KingVision, and Pentax AWS200 (92.7 s vs. 67.7 s, 66.4 s, and 62.8 s, respectively; $p<0.001, p<0.001$, and $p<0.001$, respectively). It was also longer with VividTrac than with Pentax AWS200 ${ }^{\mathrm{TM}}$ (82.9 vs. $62.8 \mathrm{~s} ; p=0.0028$ ). There was no difference in laryngoscopy time between direct laryngoscopy and VividTrac (92.7 vs. $82.9 \mathrm{~s} ; p=0.12)$.

\section{Glottic visualisation}

Glottic visualisation, as assessed by the POGO score, was poorer with direct laryngoscopy than with the four videolaryngoscopes (57.1 vs. 85.5, 91.0, 89.7 and 79.3 for Airtraq, KingVision, Pentax AWS200, and VividTrac, respectively; $p<0.001$ for all four comparisons). The results were similar when the experience of the participants was taken into account (Table 3). Glottic visualisation was poorer with VividTrac compared to KingVision and Pentax AWS200 (79.3 vs. 91.0 and 89.7, respectively; $p<0.0001$ and $p=0.0003$, respectively).

\section{Ease of use}

Ease of use of the device as rated by the participants was lower with the Macintosh blade than with Airtraq ${ }^{\mathrm{TM}}$, KingVision $^{\mathrm{TM}}$, and Pentax AWS200 ${ }^{\mathrm{TM}}$ (6.35 vs. 7.09, 7.41, and 7.93, respectively; $p<0.0001$ for all three comparisons, Table 2). There was no difference between the Macintosh blade and VividTrac (6.35 vs. 6.20; $p=0.66$ ). Again, the results were similar when the experience of the participants was taken into account (Table 3).

\section{Discussion}

In this study, three of the four videolaryngoscopes, KingVision $^{\mathrm{TM}}$, Pentax AWS200 ${ }^{\mathrm{TM}}$, and Airtraq ${ }^{\mathrm{TM}}$, met our predefined target of a lower $95 \%$ CI boundary of $90 \%$ or more for the proportion of successful first-pass attempts. With all four videolaryngoscopes, the first-pass success rate was significantly higher than with direct laryngoscopy using a Macintosh blade.

A comparative mannikin study found that videolaryngoscopy with a Macintosh-like blade performed better than did channelled videolaryngoscopy for the normal airway, whereas the opposite was true for difficult airways [32]. In a study that used a mannikin with a normal airway, direct laryngoscopy was fastest and also provided a higher first-pass success rate than did the Airtraq $^{\mathrm{TM}}$ in the hands of non-experts [33]. These differences with our results are due to the lower proportion of first-pass success with direct laryngoscopy intubation in 
Table 1 Participant characteristics

\begin{tabular}{|c|c|}
\hline Characteristics & Description $(n=79)$ \\
\hline \multicolumn{2}{|l|}{ Area of training, $n(\%)$} \\
\hline Emergency medicine & $24(30 \%)$ \\
\hline Anesthesiology and intensive care & $40(51 \%)$ \\
\hline Other & $15(19 \%)$ \\
\hline Number of semesters of residency, median [IQR] & $3[1 ; 5]$ \\
\hline \multicolumn{2}{|l|}{ Prior experience with direct laryngoscopy on a manikin } \\
\hline 0 & $4(5 \%)$ \\
\hline $1-4$ & $24(31 \%)$ \\
\hline $5-9$ & $31(40 \%)$ \\
\hline $10-19$ & $18(23 \%)$ \\
\hline$\geq 20$ & $1(1 \%)$ \\
\hline \multicolumn{2}{|l|}{ Prior experience with direct laryngoscopy on patients } \\
\hline \multicolumn{2}{|l|}{ Qualified as non-expert } \\
\hline 0 & $4(5 \%)$ \\
\hline $1-4$ & $12(15 \%)$ \\
\hline $5-9$ & $6(8 \%)$ \\
\hline $10-19$ & $16(20 \%)$ \\
\hline $20-49$ & $16(20 \%)$ \\
\hline \multicolumn{2}{|l|}{ Qualified as Expert } \\
\hline$\geq 50$ & $25(32 \%)$ \\
\hline \multicolumn{2}{|c|}{ Prior experience with videolaryngoscopy on a mannikin } \\
\hline None & $20(25 \%)$ \\
\hline$\geq 1$ & $59(75 \%)$ \\
\hline \multicolumn{2}{|l|}{ Prior experience with videolaryngoscopy on patients } \\
\hline None & $33(42 \%)$ \\
\hline$\geq 1$ & $46(58 \%)$ \\
\hline \multicolumn{2}{|c|}{ Prior experience with videolaryngoscopy without a guiding channel on patients } \\
\hline 0 & $40(51 \%)$ \\
\hline $1-4$ & $19(24 \%)$ \\
\hline $5-9$ & $10(13 \%)$ \\
\hline $10-19$ & $8(10.1 \%)$ \\
\hline$\geq 20$ & $2(2.5 \%)$ \\
\hline \multicolumn{2}{|c|}{ Prior experience with videolaryngoscopy with a guiding channel on patients } \\
\hline 0 & $52(66 \%)$ \\
\hline $1-4$ & $21(26.6 \%)$ \\
\hline $5-9$ & $3(3.8 \%)$ \\
\hline $10-19$ & $3(3.8 \%)$ \\
\hline$\geq 20$ & $0(0 \%)$ \\
\hline
\end{tabular}

our trial compared to previous studies: first-pass success rates ranged from 79 to $100 \%$ with direct laryngoscopy, even in the hands of non-experts, [32-35] compared to $71 \%$ in our study. The first-pass success rates for the videolaryngoscopes, in contrast, were comparable, at about $83-100 \%$ [32-34]. We suggest several hypotheses to explain these differences. First, intubation was made more difficult in our study by inflating the tongue to reduce the size of the pharynx and to impair glottic visualisation. We made this choice, because the proportion of successful first-pass intubations with videolaryngoscopy performed by inexperienced operators on ICU patients was $67.7 \%$ in the MACMAN1 trial [10]. Two of the above-mentioned studies on mannikins used 


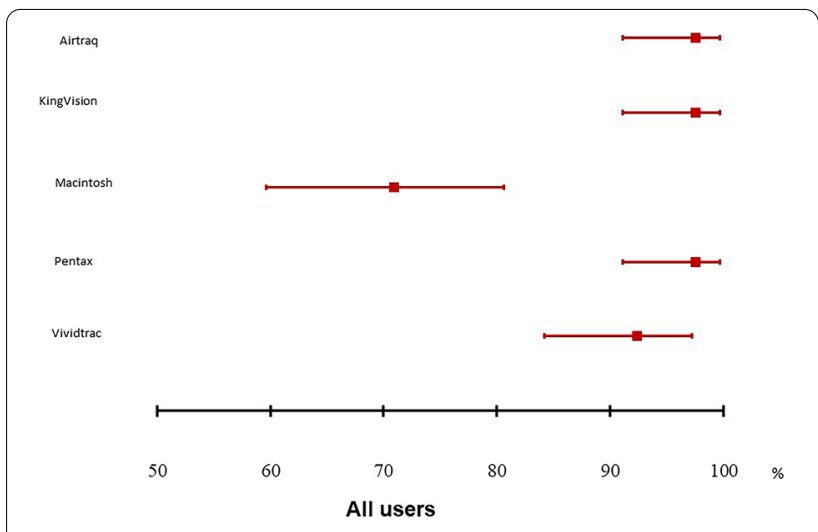

Fig. 2 Forest plot of the first-pass success rate with each videolaryngoscope included in the MACMAN2 trial

only a normal airway $[33,35]$. The mean POGO score by direct laryngoscopy ranged from 77 to 80 in the two studies reporting this variable [32, 34], both of which simulated difficult airways, compared with 57 in our study. Second, the use of a stylet or bougie to guide the tube was not allowed in our study for the first attempt, despite the simulated difficult airway. Using a stylet or bougie is recommended for the first attempt when the intubation is predicted to be difficult and secondarily if the first attempt fails [31]. For direct laryngoscopy intubation, a recent study found that a bougie was superior over a short stylet [15] and another study that a stylet was superior over no stylet [37]. If the first attempt failed, the participant could request a bougie in the present study, as happened for $40.5 \%$ of the direct laryngoscopy intubations. Finally, the previous studies did not recreate a scenario that replicated intubation of a critically ill patient in the ICU. We were able to use data from the MACMAN1 trial to replicate the conditions of intubation in critical

Table 2 Comparison of the five devices

\begin{tabular}{|c|c|c|c|c|c|c|}
\hline & Macintosh $n=79$ & AirTraq $n=79$ & KingVision $n=79$ & Pentax AWS200 $n=79$ & VividTrac $n=79$ & $p$ value \\
\hline First-pass success & $56(70.8 \%)$ & 77 (97.5\%) & $77(97.5 \%)$ & 77 (97.5\%) & $73(92.4 \%)$ & $<0.001$ \\
\hline \multicolumn{7}{|l|}{$\begin{array}{l}\text { Number of attempts before } \\
\text { success }\end{array}$} \\
\hline 1 & $56(70.8 \%)$ & 77 (97.5\%) & $77(97.5 \%)$ & 77 (97.5\%) & 73 (92.4\%) & \multirow[t]{4}{*}{$<0.001$} \\
\hline 2 & $11(14.0 \%)$ & $0(0 \%)$ & $2(2.5 \%)$ & $2(2.5 \%)$ & $1(1.3 \%)$ & \\
\hline 3 & $11(14.0 \%)$ & $1(1.3 \%)$ & $0(0 \%)$ & $0(0 \%)$ & $4(6.3 \%)$ & \\
\hline 4 & $1(1.2 \%)$ & $0(0 \%)$ & $0(0 \%)$ & $0(0 \%)$ & $0(0 \%)$ & \\
\hline $\begin{array}{l}\text { Time to intubation, s, mean } \pm \\
\text { SD }\end{array}$ & $92.7 \pm 59.8$ & $67.7 \pm 30.7$ & $66.4 \pm 24.1$ & $62.8 \pm 33.9$ & $82.9 \pm 42.8$ & $<0.001$ \\
\hline Lowest $\mathrm{SpO}_{2}$, median [IQR] & 86 [81-89] & 88 [87-90] & 88 [86-89] & 89 [87-90] & 86 [85-89] & $\$$ \\
\hline Bougie use & $32(40.5 \%)$ & $0(0 \%)$ & $0(0 \%)$ & $0(0 \%)$ & $0(0 \%)$ & $<0.001$ \\
\hline BURP manoeuvre & $18(22.8 \%)$ & $0(0 \%)$ & $0(0 \%)$ & $0(0 \%)$ & $1(1.3 \%)$ & * \\
\hline $\begin{array}{l}\text { Percentage glottic opening, } \\
\text { mean } \pm \mathrm{SD}\end{array}$ & $57.1 \pm 25.9 \%$ & $85.5 \pm 15.2 \%$ & $91.0 \pm 13.3 \%$ & $89.7 \pm 13.5 \%$ & $79.3 \pm 20.9 \%$ & $<0.001$ \\
\hline \multicolumn{7}{|l|}{ Cormack-Lehane grade } \\
\hline 1 & $23(30 \%)$ & $69(90 \%)$ & $72(95 \%)$ & 71 (91\%) & $62(80 \%)$ & \multirow[t]{4}{*}{$<0.001$} \\
\hline 2 & $39(51 \%)$ & $8(10 \%)$ & $4(5 \%)$ & $7(9 \%)$ & $15(19 \%)$ & \\
\hline 3 & $13(16 \%)$ & $0(0 \%)$ & $0(0 \%)$ & $0(0 \%)$ & $0(0 \%)$ & \\
\hline 4 & $2(3 \%)$ & $0(0 \%)$ & $0(0 \%)$ & $0(0 \%)$ & $1(1 \%)$ & \\
\hline Ease of device use $\mathrm{a}^{\mathrm{a}}$ & $6.35 \pm 2.20$ & $7.09 \pm 1.59$ & $7.41 \pm 1.59$ & $7.93 \pm 1.47$ & $6.20 \pm 2.05$ & $<0.001$ \\
\hline Oesophageal intubation & $10(12.66 \%)$ & $1(1.27 \%)$ & $1(1.28 \%)$ & $0(0 \%)$ & $0(0 \%)$ & * \\
\hline Selective intubation & $6(7.59 \%)$ & $2(2.53 \%)$ & $3(3.8 \%)$ & $5(6.3 \%)$ & $5(6.3 \%)$ & * \\
\hline $\begin{array}{l}\text { Blade placed under the epiglot- } \\
\text { tis }\end{array}$ & $8(10 \%)$ & $56(71 \%)$ & $29(37 \%)$ & 79 (100\%) & $64(81 \%)$ & $<0.001$ \\
\hline
\end{tabular}

The data are the mean \pm SD or number (\%)

BURP backwards, upwards, and rightwards pressure applied to the larynx

${ }^{\text {a }}$ Rated by the participants on a scale from 0 to 10

* The number of events was too small to allow a meaningful analysis

${ }^{\$}$ Not compared as $\mathrm{SpO}_{2}$ correlates with intubation time in a mannikin study 
Table 3 Comparison of the five devices used by participants who were inexperienced $(n=54)$ vs. experienced ( $n=25)$ with orotracheal intubation in critical care

\begin{tabular}{|c|c|c|c|c|c|c|}
\hline Groups & Macintosh $n=79$ & AirTraq $n=79$ & KingVision $n=79$ & $\begin{array}{l}\text { Pentax AWS200 } \\
n=79\end{array}$ & VividTrac $n=79$ & $p$ value \\
\hline \multicolumn{7}{|l|}{$\begin{array}{l}\text { First-attempt suc- } \\
\text { cess }\end{array}$} \\
\hline Experienced & $18(72.0)$ & $25(100.0)$ & $25(100.0)$ & $24(96.0)$ & $23(92.0)$ & $<0.001$ \\
\hline Inexperienced & $38(70.0)$ & $52(96.3)$ & $52(96.3)$ & $53(98.2)$ & $50(92.6)$ & $<0.001$ \\
\hline \multicolumn{7}{|l|}{$\begin{array}{l}\text { Time to intuba- } \\
\text { tion (s) }\end{array}$} \\
\hline Experienced & $86.73(57.3)$ & $55.41(16.4)$ & $56.55(25.5)$ & $50.44(20.7)$ & $78.16(45.8)$ & $<0.001$ \\
\hline Inexperienced & $95.46(61.3)$ & $73.56(34.2)$ & $70.97(22.3)$ & $68.52(37.4)$ & $85.14(41.5)$ & 0.002 \\
\hline \multicolumn{7}{|l|}{ Lowest $\mathrm{SpO}_{2}(\%)$} \\
\hline Experienced & $85.33(5.21)$ & 88.09 (1.78) & $88.13(2.87)$ & $88.74(2.14)$ & $87.13(4.36)$ & 0.01 \\
\hline Inexperienced & $84.02(7.39)$ & 86.88 (3.74) & $87.12(2.14)$ & $87.48(3.75)$ & $85.70(3.75)$ & $<0.001$ \\
\hline \multicolumn{7}{|c|}{$\begin{array}{l}\text { Percentage of glot- } \\
\text { tic opening }\end{array}$} \\
\hline Experienced & $56.88(29.4)$ & $88.96(13.0)$ & $90.42(13.9)$ & 88.54 (13.6) & 76.04 (19.6) & $<0.001$ \\
\hline Inexperienced & $57.25(24.4)$ & $83.98(15.9)$ & $91.30(13.2)$ & 90.19 (13.6) & $80.74(21.4)$ & $<0.001$ \\
\hline \multicolumn{7}{|l|}{ Ease of device use } \\
\hline Experienced & $6.92(1.93)$ & 7.29 (1.73) & $7.83(1.63)$ & 8.00 (1.79) & $5.67(1.74)$ & $<0.001$ \\
\hline Inexperienced & $6.08(2.29)$ & $7.00(1.53)$ & $7.21(1.55)$ & $7.90(1.32)$ & $6.44(2.15)$ & $<0.001$ \\
\hline
\end{tabular}

The data are the mean \pm SD or number (\%)

${ }^{a}$ Rated by the participants on a scale from 0 to 10

care, which are associated with an increased frequency of complications and a decreased first-pass success rate.

Few studies have compared different types of channelled videolaryngoscope. Outside the setting of critical care, a study of 720 patients undergoing elective surgery used a cervical collar to increase intubation difficulty. All intubations were performed by senior anaesthesiologists. Three channelled videolaryngoscopes were used: Airtraq and KingVision were associated with first-pass success rates of 85 and $87 \%$, respectively, compared to only $37 \%$ with A.P. Advance ${ }^{\mathrm{TM}}$ [26]. We found lower first-pass success rates with VividTrac compared to the other three videolaryngoscopes, although the difference was not statistically significant. In another study, the intubation time was longer with VividTrac than with KingVision [36]. In a comparison of KingVision, Airtraq, and VividTrac that used an airway trainer with normal and difficult airway options and included medical students with no intubation experience, the only significant difference was a higher first-pass success rate with VividTrac than with KingVision for the difficult airway (100 vs. 92\%, respectively; $p<0.05$ ) [34]. In contrast, in our study, VividTrac was the only channelled videolaryngoscope for which the lower boundary of the 95\% CI for the first-pass success rate was below $90 \%$. Furthermore, the duration of laryngoscopy was significantly longer and glottic visualisation poorer compared to the other three videolaryngoscopes. The participants also gave the VividTrac lower ease-ofuse ratings compared to the other devices.

In our study, the first-pass success rate was significantly higher with the videolaryngoscopes than with direct laryngoscopy for both experienced and inexperienced intubators. The differences between the videolaryngoscopes and direct laryngoscopy in terms of laryngoscopy time, glottic visualisation, and ease of use were also similar in the two participant groups. This finding is somewhat surprising, since a higher level of experience with intubation has been shown to correlate with a higher first-pass success rate when performing emergency ETI in critically ill patients [37, 38]. Furthermore, in a metaanalysis focussing on ETI outside the operating room, first-pass intubation was more common with a videolaryngoscope than with direct laryngoscopy among the novices (81.4 vs. $71.5 \%$; odds ratio, 1.95; 95\% CI, 1.45-2.64; $p<0.001$ ) but not among the experienced intubators [20]. However, novices were variably defined in the different studies included in this work. The shorter learning curve for videolaryngoscopy than for direct laryngoscopy may explain that novices do better with the former [39]. Thus, having performed only 15 or more videolaryngoscopies was independently associated with first-pass success in critically ill patients [39]. 
We used 50 intubations as the cutoff to differentiate inexperienced from experienced intubators based on a metaanalysis showing that, above this level of experience, the success rate of one or two attempts was greater than $90 \%$ [38]. Most of our participants had limited experience with intubation, and the residents most likely to acquire such experience, i.e., those in emergency medicine and intensive care, were only in their first or second year of residency. All participants would have met the definition of non-experts used in the MACMAN trial (5 years in ICUs or 2 years in anaesthesia and 1 year in ICUs) [38]. We selected these participants, because novice intubators perform the first intubation attempt in more than $80 \%$ of patients in ICUs in France [10].

Our trial has several limitations. First, extrapolation from results obtained using a mannikin to those in patients requires circumspection. Airway anatomy is among the major determinants of intubation difficulty and, despite recent advances, mannikins do not accurately replicate the human airway anatomy. For instance, by computed tomography, the upper airway size of the SimMan $3 G^{\circledR}$ was larger than that of patients [40]. Soft tissue elasticity is an important feature during direct laryngoscopy and was not accurately replicated by our mannikin. Other details were not present in the simulation, such as fogging or secretions that may limit visibility during intubation. However, it is ethically preferable to perform this type of study on mannikins [41], thus protecting patients from potential complications related to videolaryngoscopy use by novices. High-fidelity simulation also makes it possible to create a difficult airway, which is not extraordinarily frequent in clinical practice, and ensures that the study environment remains unchanged for all participants and all devices [42, 43]. Second, oxygenation in the event of desaturation was not technically feasible with our setup. This probably overestimated the first-pass success rate as, in the event of a long intubation time, the participant did not stop the attempt to re-ventilate the patient. There was also no possibility of changing the intubation device for another possibly more effective one. This point no doubt increased the mean intubation time. However, the mean intubation time, which was the laryngoscopy time increased by $45 \mathrm{~s}$ to allow for induction, was lower than that found in the MACMAN 1 trial [10]. Last, we defined experts as having performed more than 50 direct laryngoscopies. This level of experience in direct laryngoscopy may not translate to a similar level with videolaryngoscopy. In an observational ICU study reported in 2020, experts were defined as having performed at least 15 videolaryngoscopy ETIs [41].

\section{Conclusion}

Among the four videolaryngoscopes tested, three had a lower boundary of the $95 \%$ CI for the first-pass success rate higher than 90\%: Airtraq $^{\mathrm{TM}}$, KingVision ${ }^{\mathrm{TM}}$, and Pentax AWS200 ${ }^{\mathrm{TM}}$. These three devices were superior over direct laryngoscopy in our model of a patient in critical condition due to acute respiratory failure. Further randomised controlled trials are required to better define the role for videolaryngoscopes and associated tools for the intubation of critically ill patients. Including the three videolaryngoscopes found efficient in the present study, and pooling them due to their similar performance, may facilitate such trials.

\section{Supplementary Information}

The online version contains supplementary material available at https://doi. org/10.1186/s13613-021-00916-3.

Additional file 1: Figure S1. Consort flow diagram template.

Additional file 2: Figure S2. Inexperienced users.

Additional file 3: Figure S3. Experienced users.

\section{Acknowledgements}

We are indebted to A. Wolfe, MD, who helped to prepare and review the manuscript.

\section{Authors' contributions}

Study design, development and study setup were performed by PD, NG, CL, $J R$, and JBL. Site setup, participant enrolment, data collection, and research governance were performed by PD and JBL. ALT performed the statistical analysis. The initial draft of the manuscript was written by PD, ALT, and JBL. All authors reviewed this and subsequent versions of the manuscript for important intellectual content. All authors vouch for the accuracy and completeness of the data and analyses and for the fidelity of the trial to the protocol. All authors read and approved the final manuscript.

Funding

Not funded.

Availability of data and materials

The study data will be made available upon reasonable request to the corresponding author.

Code availability

Not applicable.

\section{Declarations}

Ethics approval and consent to participate

The study protocol was approved by the Ethics Committee of the Société de Réanimation de Langue Française (CE SRLF 17-11). Each participant was informed at the beginning of each session about the objectives of the trial, the recording of the simulation sessions, and her or his right to access and refuse the use of the data collected for the trial.

Consent for publication

Not applicable. 


\section{Competing interests}

None of the authors has any competing interests to declare.

\section{Author details}

${ }^{1}$ Médecine Intensive Réanimation, Centre Hospitalier Universitaire de Nantes, Nantes, France. ${ }^{2}$ Service d'Anesthésie Réanimation Chirurgicale, Université de Nantes, CHU Nantes, Pôle Anesthésie-Réanimation, Hôtel Dieu, 44093 Nantes, France. ${ }^{3}$ Laboratoire Expérimental de Simulation de Médecine Intensive de L'Université (LE SiMU) de Nantes, 9 rue Bias, 44001 Nantes, France. ${ }^{4}$ Plateforme de Méthodologie Et Biostatistique, Direction de La Recherche de L'Innovation, Centre Hospitalier Universitaire de Nantes, Nantes, France. ${ }^{5}$ Médecine Intensive Réanimation, Centre Hospitalier Universitaire de Nantes, Nantes, France.

Received: 7 May 2021 Accepted: 4 August 2021

Published online: 16 August 2021

\section{References}

1. Heuer JF, Barwing TA, Barwing J, Russo SG, Bleckmann E, Quintel M, et al. Incidence of difficult intubation in intensive care patients: analysis of contributing factors. Anaesth Intensiv Care. 2012;40(1):120-7.

2. Jaber S, Jung B, Corne P, Sebbane M, Muller L, Chanques G, et al. An intervention to decrease complications related to endotracheal intubation in the intensive care unit: a prospective, multiple-center study. Intensiv Care Med. 2010;36(2):248-55.

3. Russotto V, Myatra SN, Laffey JG, Tassistro E, Antolini L, Bauer P, et al. Intubation practices and adverse peri-intubation events in critically ill patients from 29 countries. JAMA. 2021;325(12):1164-72.

4. De Jong A, Rolle A, Molinari N, Paugam-Burtz C, Constantin JM, Lefrant $J Y$, et al. Cardiac arrest and mortality related to intubation procedure in critically ill adult patients: a multicenter cohort study. Crit Care Med. 2018;46(4):532-9.

5. Griesdale DE, Bosma TL, Kurth T, Isac G, Chittock DR. Complications of endotracheal intubation in the critically ill. Intensiv Care Med. 2008;34(10):1835-42.

6. Mort TC. Emergency tracheal intubation: complications associated with repeated laryngoscopic attempts. Anesth Analg. 2004;99(2):607-13.

7. Sakles JC, Chiu S, Mosier J, Walker C, Stolz U. The importance of first pass success when performing orotracheal intubation in the emergency department. Acad Emerg Med. 2013;20(1):71-8.

8. De Jong A, Molinari N, Terzi N, Mongardon N, Arnal JM, Guitton C, et al. Early identification of patients at risk for difficult intubation in the intensive care unit: development and validation of the MACOCHA score in a multicenter cohort study. Am J Respir Crit Care Med. 2013;187(8):832-9.

9. Schwartz DE, Matthay MA, Cohen NH. Death and other complications of emergency airway management in critically ill adults. A prospective investigation of 297 tracheal intubations. Anesthesiology. 1995;82(2):367-76.

10. Lascarrou JB, Boisrame-Helms J, Bailly A, Le Thuaut A, Kamel T, Mercier E, et al. Video laryngoscopy vs direct laryngoscopy on successful first-pass orotracheal intubation among icu patients: a randomized clinical trial. JAMA. 2017:317(5):483-93.

11. Guitton C, Ehrmann S, Volteau C, Colin G, Maamar A, Jean-Michel V, et al. Nasal high-flow preoxygenation for endotracheal intubation in the critically ill patient: a randomized clinical trial. Intensiv Care Med. 2019;45(4):447-58.

12. Jaber S, De Jong A, Pelosi P, Cabrini L, Reignier J, Lascarrou JB. Videolaryngoscopy in critically ill patients. Crit Care. 2019;23(1):221.

13. Martin M, Decamps P, Seguin A, Garret C, Crosby L, Zambon O, et al. Nationwide survey on training and device utilization during tracheal intubation in French intensive care units. Ann Intensive Care. 2020;10(1):2.

14. CookTM, Kelly FE. A national survey of videolaryngoscopy in the United Kingdom. Br J Anaesth. 2017;118(4):593-600.

15. Driver BE, Prekker ME, Klein LR, Reardon RF, Miner JR, Fagerstrom ET, et al. Effect of use of a bougie vs endotracheal tube and stylet on first-attempt intubation success among patients with difficult airways undergoing emergency intubation: a randomized clinical trial. JAMA. 2018:319(21):2179-89.

16. Murakami Y, Ueki R, Niki M, Hirose M, Shimode N. Three-day tracheal intubation manikin training for novice doctors using Macintosh laryngoscope, McGRATH MAC videolaryngoscope and Pentax AirwayScope. Medicine. 2021;100(4):e23886.

17. Mosier JM, Whitmore SP, Bloom JW, Snyder LS, Graham LA, Carr GE, et al. Video laryngoscopy improves intubation success and reduces esophageal intubations compared to direct laryngoscopy in the medical intensive care unit. Crit Care. 2013;17(5):R237.

18. De Jong A, Clavieras N, Conseil M, Coisel Y, Moury PH, Pouzeratte $Y$, et al. Implementation of a combo videolaryngoscope for intubation in critically ill patients: a before-after comparative study. Intensiv Care Med. 2013;39(12):2144-52.

19. Noppens RR, Geimer S, Eisel N, David M, Piepho T. Endotracheal intubation using the C-MAC(R) video laryngoscope or the Macintosh laryngoscope: a prospective, comparative study in the ICU. Crit Care. 2012;16(3):R103.

20. Arulkumaran N, Lowe J, lons R, Mendoza M, Bennett V, Dunser MW. Videolaryngoscopy versus direct laryngoscopy for emergency orotracheal intubation outside the operating room: a systematic review and metaanalysis. Br J Anaesth. 2018;120(4):712-24.

21. Gao YX, Song YB, Gu ZJ, Zhang JS, Chen XF, Sun H, et al. Video versus direct laryngoscopy on successful first-pass endotracheal intubation in ICU patients. World J Emerg Med. 2018;9(2):99-104.

22. Huang HB, Peng JM, Xu B, Liu GY, Du B. Video laryngoscopy for endotracheal intubation of critically ill adults: a systemic review and meta-analysis. Chest. 2017:152(3):510-7.

23. Akihisa Y, Maruyama K, Koyama Y, Yamada R, Ogura A, Andoh T. Comparison of intubation performance between the King Vision and Macintosh laryngoscopes in novice personnel: a randomized, crossover manikin study. J Anesth. 2014;28(1):51-7.

24. Lee J, Kwak HJ, Lee JY, Chang MY, Lee SY, Kim JY. Comparison of the Pentax AirwayScope and MCGrath MAC videolaryngoscope for endotracheal intubation in patients with a normal airway. Medicine. 2017;96(46):e8713.

25. Mendonca C, Ungureanu N, Nowicka A, Kumar P. A randomised clinical trial comparing the "sniffing" and neutral position using channelled $\left(\right.$ KingVision $\left.^{\circledR}\right)$ and non-channelled (C-MAC $\left.{ }^{\circledR}\right)$ videolaryngoscopes. Anaesthesia. 2018;73(7):847-55.

26. Kleine-Brueggeney M, Greif R, Schoettker P, Savoldelli GL, Nabecker S, Theiler LG. Evaluation of six videolaryngoscopes in 720 patients with a simulated difficult airway: a multicentre randomized controlled trial. $\mathrm{Br}$ J Anaesth. 2016;116(5):670-9.

27. Bailly A, Lascarrou JB, Le Thuaut A, Boisrame-Helms J, Kamel T, Mercier E, et al. McGRATH MAC videolaryngoscope versus Macintosh laryngoscope for orotracheal intubation in intensive care patients: the randomised multicentre MACMAN trial study protocol. BMJ Open. 2015. https://doi. org/10.1136/bmjopen-2015-009855.

28. Bailly A, Ricard JD, Le Thuaut A, Helms J, Kamel T, Mercier E, et al. Compared efficacy of four preoxygenation methods for intubation in the ICU: retrospective analysis of McGrath Mac videolaryngoscope versus Macintosh laryngoscope (MACMAN) trial data. Crit Care Med. 2019. https://doi. org/10.1097/CCM.0000000000003656.

29. McCrory JW, Matthews JN. Comparison of four methods of preoxygenation. Br J Anaesth. 1990;64(5):571-6.

30. Higgs A, McGrath BA, Goddard C, Rangasami J, Suntharalingam G, Gale R, et al. Guidelines for the management of tracheal intubation in critically ill adults. Br J Anaesth. 2018;120(2):323-52.

31. Quintard H, I'Her E, Pottecher J, Adnet F, Constantin JM, De Jong A, et al. Experts' guidelines of intubation and extubation of the ICU patient of French Society of Anaesthesia and Intensive Care Medicine (SFAR) and French-speaking Intensive Care Society (SRLF) : In collaboration with the pediatric Association of French-speaking Anaesthetists and Intensivists (ADARPEF), French-speaking Group of Intensive Care and Paediatric emergencies (GFRUP) and Intensive Care physiotherapy society (SKR). Ann Intensiv Care. 2019;9(1):13.

32. De Jong A, Rolle A, Pensier J, Capdevila M, Jaber S. First-attempt success is associated with fewer complications related to intubation in the intensive care unit. Intensiv Care Med. 2020;46(6):1278-80.

33. Eismann $H$, Sieg L, Etti N, Friedrich L, Schröter C, Mommsen P, et al. Improved success rates using videolaryngoscopy in unexperienced users: a randomized crossover study in airway manikins. Eur J Med Res. 2017;22(1):27

34. Pieters BMA, Wilbers NER, Huijzer M, Winkens B, van Zundert AAJ. Comparison of seven videolaryngoscopes with the Macintosh laryngoscope 
in manikins by experienced and novice personnel. Anaesthesia. 2016;71(5):556-64.

35. Rendeki S, Keresztes D, Woth G, Mérei Á, Rozanovic M, Rendeki M, et al. Comparison of VividTrac ${ }^{\circledR}$, Airtraq $^{\circledR}$, King Vision $^{\circledR}$, Macintosh laryngoscope and a custom-made videolaryngoscope for difficult and normal airways in mannequins by novices. BMC Anesthesiol. 2017;17(1):68.

36. Okada D, Komasawa N, Fujiwara S, Minami T. Comparison of tubeguided and guideless videolaryngoscope for tracheal intubation during chest compression in a manikin: a randomized crossover trial. J Anesth. 2015;29(3):331-7.

37. Jaber S, Rollé A, Godet T, Terzi N, Riu B, Asfar P, et al. Effect of the use of an endotracheal tube and stylet versus an endotracheal tube alone on first-attempt intubation success: a multicentre, randomised clinical trial in 999 patients. Intensiv Care Med. 2021;47(6):653-64

38. Martínez AH, García JMA, Rosete VAM. Intubation time between videolaryngoscopes: King Vision versus Vivid Trac. Comp Study Acta Médica Grupo Ángeles. 2016;14(3):131-5.

39. Hirsch-Allen AJ, Ayas N, Mountain S, Dodek P, Peets A, Griesdale DE. Influence of residency training on multiple attempts at endotracheal intubation. Can J Anaesth. 2010;57(9):823-9.
40. Simpson GD, Ross MJ, McKeown DW, Ray DC. Tracheal intubation in the critically ill: a multi-centre national study of practice and complications. Br J Anaesth. 2012;108(5):792-9.

41. Amalric M, Larcher R, Brunot V, Garnier F, De Jong A, Moulaire Rigollet V, et al. Impact of videolaryngoscopy expertise on first-attempt intubation success in critically ill patients. Crit Care Med. 2020;48(10):e889-96.

42. Schebesta K, Hüpfl M, Rössler B, Ringl H, Müller MP, Kimberger O. Degrees of reality: airway anatomy of high-fidelity human patient simulators and airway trainers. Anesthesiology. 2012;116(6):1204-9.

43. Rai MR, Popat MT. Evaluation of airway equipment: man or manikin? Anaesthesia. 2011;66(1):1-3.

\section{Publisher's Note}

Springer Nature remains neutral with regard to jurisdictional claims in published maps and institutional affiliations.

\section{Submit your manuscript to a SpringerOpen ${ }^{\circ}$ journal and benefit from:}

- Convenient online submission

- Rigorous peer review

- Open access: articles freely available online

- High visibility within the field

- Retaining the copyright to your article

Submit your next manuscript at springeropen.com 